\title{
Synthesis and Characterization of New Compounds in the Series 1-alkyl-4- [2-aryl-1-diazenyl]piperazines
}

\author{
Erin MacLeod and Keith Vaughan*
}

Department of Chemistry, Saint Mary's University, Halifax, N.S., Canada

\begin{abstract}
New triazenes derived from the 1-alkylpiperazines have been synthesized. Each 1-alkylpiperazine was treated with a diazonium salt solution to produce the new triazenes, which have been characterized by proton and carbon13 NMR spectroscopy, IR spectroscopy and by mass spectrometric analysis. Assignment of the chemical shifts to specific protons and carbons in the piperazine ring was facilitated by comparison with the chemical shifts in the previously reported 1-methyl-4-[2-aryl-1-diazenyl]piperazines ().
\end{abstract}

Keywords: 1-Alkylpiperazine, aryldiazenyl, diazonium coupling, piperazine, triazene.

\section{INTRODUCTION}

In a previous report [1] a series of 1-methyl-4-[2-aryl-1diazenyl]piperazines (1) were synthesized and characterized. In this paper, we now report the formation of 24 new triazenes derived from 1-alkylpiperazines, namely the 1ethyl series (2), the 1-hydroxyethyl series (3), the 1-butyl series (4) and the 1-iso-propyl series (5).

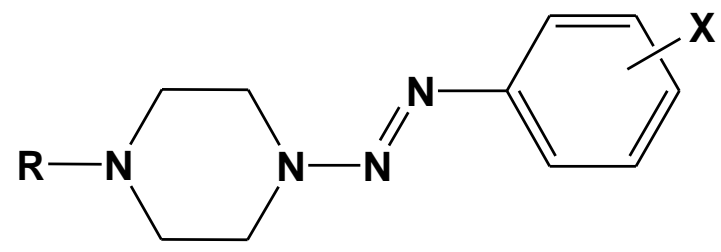

$$
\begin{aligned}
& 1 \mathrm{R}=-\mathrm{Me} \\
& 2 \mathrm{R}=-\mathrm{Et} \\
& 3 \mathrm{R}=-\mathrm{CH}_{2} \mathrm{CH}_{2} \mathrm{OH} \\
& 4 \mathrm{R}=-\mathrm{Bu} \\
& 5 \mathrm{R}=-i-\mathrm{Pr}
\end{aligned}
$$

This paper is the latest chapter in an extensive study of the synthesis of $\mathrm{N}$-aryldiazenylpiperazines and the structural characterization of these triazenes. In Part I of this series [1], the diazonium coupling reaction of 1-methylpiperazine was explored in order to synthesize the 4-methyl-1-(2-aryldiazen1-yl-)piperazines (1). "The structure of one compound, namely methyl 4-\{(E)-2-(4-methylpiperazino)-1-diazenyl $\}$ benzoate, in this series has been verified by X-ray crystallography [2], which showed that the piperazine ring adopts a normal chair conformation." In the process of characterizing these triazenes by NMR spectroscopy, the chemical shift parameters "of the protons and carbons in the piperazine ring were clearly established and verified by some

*Address correspondence to this author at the Department of Chemistry, Saint Mary's University, Halifax, N.S., Canada; Tel: (902)-420-5650; Fax: (902)-496-8104; E-mail: keith.vaughan@ @mu.ca classical 2D NMR work" [1, 2]. "This data was used to advantage in the characterization of 4-methyl-1[aryldiazenyl]-homopiperazines (ㅁ), which have been described, along with the 1,4-di-(2-aryldiazen-1-yl) diazepanes" (ㄱ), in Part II of this series [3]. The bis-triazenes (7) were obtained by reaction of homopiperazine with the diazonium salt in 1:2 molar proportion.

This bis-diazotization strategy was subsequently explored with piperazine itself and the synthesis of a series of 1,4-di(2-aryldiazen-1-yl-)-piperazines (으) was described in Part III of this series [4]. "The bis-triazenes of type $\underline{\mathbf{8}}$ were characterized by a range of spectroscopic techniques and the data was compared with some previously published work [5] in which several of the bis-aryldiazenyl-piperazines had already been described." Subsequently, we extended the study of bis-triazenes to the 1,4-di-[2-aryl-1-diazenyl]-2methylpiperazines $(\underline{\mathbf{9}})$, which were prepared by diazonium coupling to 2-methylpiperazine [6], and then to the 1,4-di-[2-

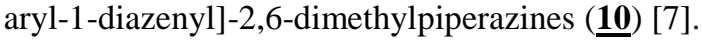

"The work described in this paper is a logical extension of our previous study [1] of the synthesis of the 1-methyl-4[2-aryl-1-diazenyl]piperazines" (1).

\section{DISCUSSION}

The reaction of a diazonium salt with 1-ethylpiperazine proceeded smoothly to afford good to excellent yields of the 1-ethyl-4-[2-aryl-1-diazenyl]piperazine series (2). Yields and physical data of these compounds are shown in Table $\mathbf{1}$. The compounds of series $\mathbf{2}$ are low-melting point solids, which have high solubility in a range of solvents, making it difficult to achieve purification by recrystallization in all cases. Some success was achieved with hexanes or ethyl acetate/hexanes mixture. The IR data show the presence of the aryl substituent functional group absorptions, as well as the appropriate OOP bands for the particular substituent in the benzene ring. The ${ }^{1} \mathrm{H}$ NMR data for series $\mathbf{2 a - 2 i}$ are presented in Table $\mathbf{2}$. The spectra of compounds $\mathbf{2 a - 2 g}$ show the typical signals for an AA'BB' pattern of a $p$-disubstituted 

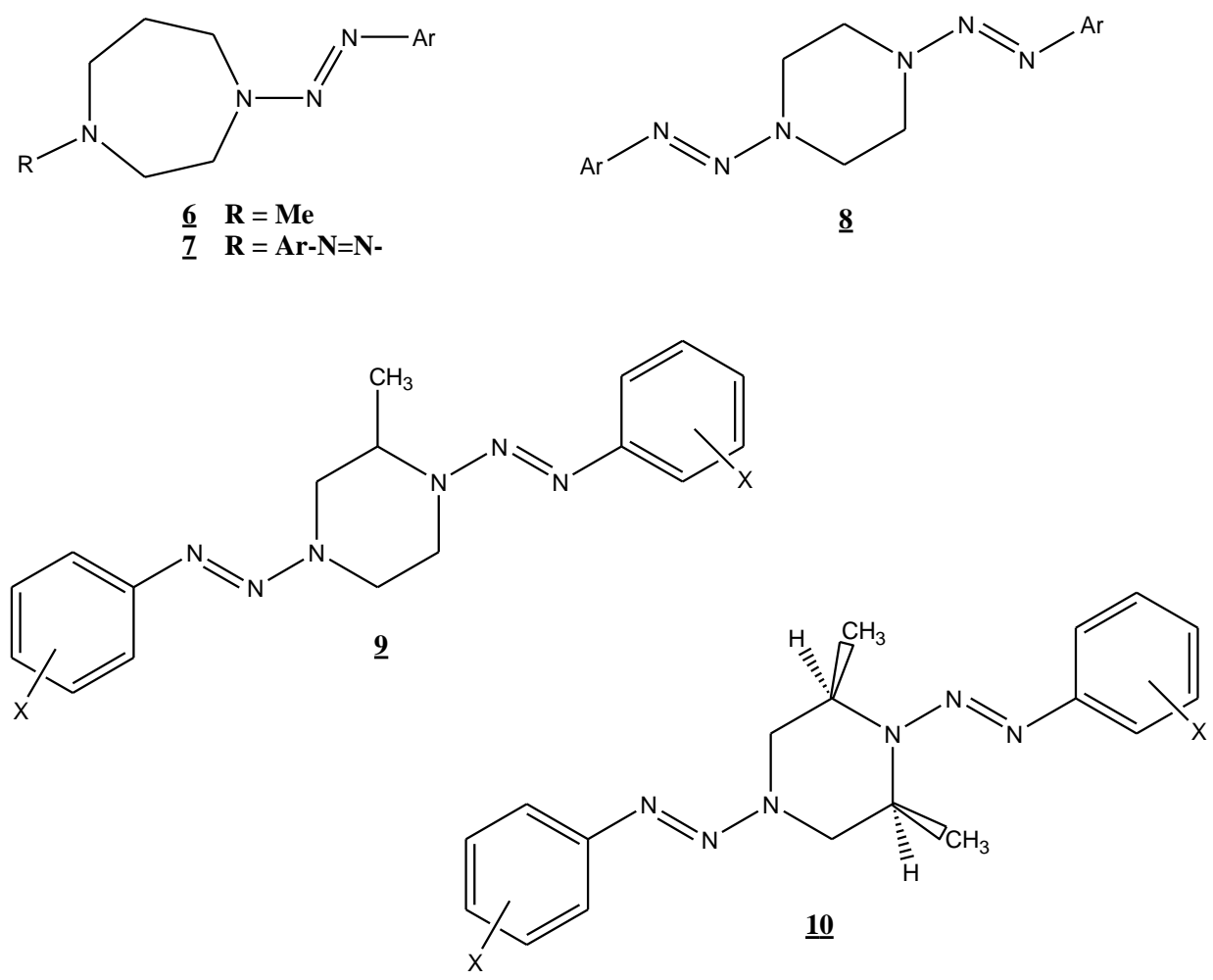

benzene derivative, whereas the $o$-bromo-derivative (2h) shows four unique chemical shifts in the aromatic region as expected with multiplicity of a doublet of doublets or doublet of triplets. The 3-pyridyl analogue (2i) also shows a pattern of four unique aromatic hydrogen atoms. All compounds in the series show the typical signals for the $\mathrm{N}$ ethyl group. i.e. a 2-proton quartet at ca. 2.48 and a 3-proton triplet at $c a$. $1.11 \mathrm{ppm}$. The protons in the piperazine ring appear as 4-proton triplets at $c a$. 2.6 and 3.8, with a coupling constant $J_{\mathrm{ab}}=c a .5 \mathrm{~Hz}$.

Extension of the diazonium coupling reaction to 1-(2hydroxyethyl)-piperazine resulted in excellent yields of the 1-(2-hydroxyethyl)-4-[2-aryl-1-diazenyl]piperazine series (3). Yields and physical data of these compounds are shown in Table 3. The compounds of this series have higher melting points than those of series $\mathbf{2}$, and they were consequently much easier to recrystallize. The ${ }^{1} \mathrm{H}$ NMR data for series 3a$\mathbf{3 i}$ are presented in Table $\mathbf{4}$. The spectra of compounds $\mathbf{3 a - 3} \mathbf{g}$ show the typical signals for an AA'BB' pattern, and the $o$ bromo- and 3-pyridyl derivatives displayed aromatic signals similar to those of $\mathbf{2 h}$ and $\mathbf{2 i}$. The protons in the piperazine ring appear as 4-proton triplets at $c a .2 .68$ and 3.85, with a coupling constant $J_{\mathrm{ab}}=c a$. $5.1 \mathrm{~Hz}$. All compounds in the series show the typical signals for the methylene groups of the 2-hydroxyethyl moiety, i.e. a 2-proton triplet at ca. 2.64 and a 2-proton triplet at ca. $3.68 \mathrm{ppm}$. The proton of the $\mathrm{OH}$ group appears in the range 1.3-1.8 ppm, with the exception of the signal at $4.82 \mathrm{ppm}$ in the 3-pyridyl derivative (3i).

Further extension of the diazonium coupling reaction to 1-butylpiperazine resulted in excellent yields of the 1-butyl4-[2-aryl-1-diazenyl]piperazine series (4). Yields and physical data of these compounds are shown in Table 5. The ${ }^{1} \mathrm{H}$ NMR data for series $\mathbf{4 a - 4 d}$ are presented in Table $\mathbf{6}$. The spectra of compounds $\mathbf{4 a - 4 d}$ show the typical signals for an AA'BB' pattern of a p-substituted aryl ring The protons in the piperazine ring appear as 4-proton triplets at ca. 2.6 and 3 , with a coupling constant $J_{a \mathrm{~b}}=c a$. $5.1 \mathrm{~Hz}$. All compounds in the series show the typical signals for the butyl group: a 2proton triplet at $c a$. 2.4, a 2-proton pentet at $c a$. 1.52, a 2proton pentet at $c a .1 .35$ and a 3-proton triplet at. $0.94 \mathrm{ppm}$. The coupling constants within the butyl group are in the range $J=c a .7 .5 \mathrm{~Hz}$.

Two compounds of the 1-isopropyl-4-[2-aryl-1diazenyl]piperazine series (5) have been synthesized. Physical data are reported in Table 7 and ${ }^{1} \mathrm{H}$ NMR data are shown in Table 8. The spectroscopic data are similar to the data of series 2, 3 and $\mathbf{4}$ and are consistent with the structures 5a and 5b. As a final proof of structure, all of the new compounds reported here have been analyzed by high resolution mass spectrometry to confirm the molecular formulas of each compound. The results for all series are tabulated in Table 9.

It is relevant to the present work that the medicinal chemistry of piperazine derivatives has attracted considerable interest. Research on arylpiperazines is quite extensive due to their biological applications. They are especially known for their high affinity toward serotonin receptors, chiefly 5-HT1A receptors. Many common anxiolytics and antidepressants incorporate arylpiperazines. It is believed that arylpiperazine agonist or antagonist activity comes from either its coplanarity or perpendicular conformation, respectively, of the aryl ring with the piperazine N1 nitrogen. The possibility of combining the structural unit of a triazene with that of a piperazine raises interesting questions like what biological activities might be generated from such a marriage. 
Table 1. Physical data of 1-ethyl-4-[2-aryl-1-diazenyl]piperazines.

\begin{tabular}{|c|c|c|c|c|c|}
\hline Compound \# & $\mathbf{X}$ & Yield & m.p. $\left({ }^{\circ} \mathbf{C}\right)(\mathbf{a})$ & Crystal Appearance & IR $\left(\mathbf{c m}^{-1}\right)$ \\
\hline \hline $2 \mathrm{a}$ & $\mathrm{p}-\mathrm{CN}$ & $84 \%$ & $57-59$ & $\begin{array}{c}\text { Glassy sheets } \\
\text { Dark Brown }\end{array}$ & $\begin{array}{c}\text { Amorphous } \\
\text { orange } \\
845.4(\mathrm{OOP})\end{array}$ \\
\hline $2 \mathrm{~b}$ & $\mathrm{p}-\mathrm{CO}_{2} \mathrm{Me}$ & $76 \%$ & $84-85$ & $\begin{array}{c}1715.4(\mathrm{C}=\mathrm{O}) \\
865 / 733(\mathrm{OOP})\end{array}$ & $\left.1511 / 1333(\mathrm{NO})_{2}\right)$ \\
\hline $2 \mathrm{c}$ & $\mathrm{p}-\mathrm{NO}_{2}$ & $60 \%$ & $87-89$ & Medium sized orange needles & $820.5(\mathrm{OOP})$ \\
\hline $2 \mathrm{~d}$ & $\mathrm{p}-\mathrm{CH}_{3}$ & $38 \%$ & $53-54$ & Amorphous Red/Orange & $616.9(\mathrm{C}-\mathrm{Br})$ \\
\hline $2 \mathrm{p}$ & $\mathrm{p}-\mathrm{Br}$ & $94 \%$ & $60-61$ & Amorphous Dark Orange & $834.5(\mathrm{OOP})$ \\
\hline $2 \mathrm{f}$ & $\mathrm{p}-\mathrm{CH}_{3} \mathrm{O}$ & $26 \%$ & $44-47$ & Amorphous Red & $1251.1\left(\mathrm{CH} \mathrm{H}_{3} \mathrm{O}\right)$ \\
$830.2(\mathrm{OOP})$
\end{tabular}

Recrystallization solvent $=$ hexanes or hexanes/ethyl acetate mixture

Table 2. ${ }^{1} \mathrm{H}$ NMR chemical shift data (ppm) in $\mathrm{CDCl}_{3}$ of the 1-ethyl-4-[2-aryl-1-diazenyl]piperazines.

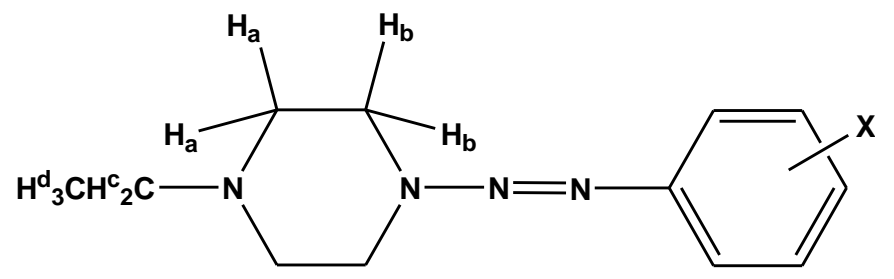

\begin{tabular}{|c|c|c|c|c|c|c|c|}
\hline Compound \# & $\mathbf{X}$ & Aromatic & $\mathbf{H}_{\mathbf{a}}$ & $\mathbf{H}_{\mathbf{b}}$ & $\mathbf{H}_{\mathrm{c}}$ & $\mathbf{H}_{\mathbf{d}}$ & $\mathbf{X}$ \\
\hline $2 \mathrm{a}$ & $\mathrm{p}-\mathrm{CN}$ & $\begin{array}{l}7.75,7.72 \\
7.56,7.53\end{array}$ & $\begin{array}{c}3.88 \\
(\mathrm{t}, 4 \mathrm{H}) \\
\mathrm{J}=5.0\end{array}$ & $\begin{array}{c}2.59 \\
\text { (broad s, 4H) }\end{array}$ & $\begin{array}{c}2.46(\mathrm{q}, 2 \mathrm{H}) \\
\mathrm{J}=6.7\end{array}$ & $1.10(\mathrm{t}, 3 \mathrm{H})$ & $\mathrm{n} / \mathrm{a}$ \\
\hline $2 b$ & $\mathrm{p}-\mathrm{CO}_{2} \mathrm{Me}$ & $\begin{array}{l}8.00,7.98 \\
7.45,7.44\end{array}$ & $\begin{array}{c}3.86 \\
(\mathrm{t}, 4 \mathrm{H}) \\
\mathrm{J}=5.4\end{array}$ & $\begin{array}{c}2.58 \\
(\mathrm{t}, 4 \mathrm{H}) \\
\mathrm{J}=4.9\end{array}$ & $\begin{array}{c}2.47(\mathrm{q}, 2 \mathrm{H}) \\
\mathrm{J}=7.2\end{array}$ & $\begin{array}{c}1.11(\mathrm{t}, 3 \mathrm{H}) \\
\mathrm{J}=7.2\end{array}$ & $\begin{array}{c}3.87 \\
(\mathrm{~s}, 3 \mathrm{H})\end{array}$ \\
\hline $2 \mathrm{c}$ & $\mathrm{p}-\mathrm{NO}_{2}$ & $\begin{array}{l}8.17,8.15 \\
7.48,7.47\end{array}$ & $\begin{array}{c}3.94 \\
\text { (broad s, 4H) }\end{array}$ & $\begin{array}{c}2.63 \\
\text { (broad s, } 4 \mathrm{H})\end{array}$ & $\begin{array}{c}2.52(\mathrm{q}, 2 \mathrm{H}) \\
\mathrm{J}=7.2\end{array}$ & $\begin{array}{c}1.13(\mathrm{t}, 3 \mathrm{H}) \\
\mathrm{J}=7.2\end{array}$ & $\mathrm{n} / \mathrm{a}$ \\
\hline $2 \mathrm{~d}$ & $\mathrm{p}-\mathrm{CH}_{3}$ & $\begin{array}{l}7.36,7.34 \\
7.15,7.13\end{array}$ & $\begin{array}{c}3.79 \\
(\mathrm{t}, 4 \mathrm{H}) \\
\mathrm{J}=5.2\end{array}$ & $\begin{array}{c}2.59 \\
(\mathrm{t}, 4 \mathrm{H}) \\
\mathrm{J}=5.2\end{array}$ & $\begin{array}{c}2.48(\mathrm{q}, 2 \mathrm{H}) \\
\mathrm{J}=7.2\end{array}$ & $\begin{array}{c}1.13(\mathrm{t}, 3 \mathrm{H}) \\
\mathrm{J}=7.2\end{array}$ & $\begin{array}{c}2.33 \\
(\mathrm{~s}, 3 \mathrm{H})\end{array}$ \\
\hline $2 \mathrm{f}$ & $\mathrm{p}-\mathrm{CH}_{3} \mathrm{O}$ & $\begin{array}{l}7.41,7.39 \\
6.88,6.86\end{array}$ & $\begin{array}{c}3.75 \\
(\mathrm{t}, 4 \mathrm{H}) \\
\mathrm{J}=5.2\end{array}$ & $\begin{array}{c}2.58 \\
(\mathrm{t}, 4 \mathrm{H}) \\
\mathrm{J}=5.3\end{array}$ & $\begin{array}{c}2.47(q, 2 \mathrm{H}) \\
\mathrm{J}=7.2\end{array}$ & $\begin{array}{c}1.12(\mathrm{t}, 3 \mathrm{H}) \\
\mathrm{J}=7.2\end{array}$ & $\begin{array}{c}3.79 \\
(\mathrm{~s}, 3 \mathrm{H})\end{array}$ \\
\hline
\end{tabular}


Table 2. Contd.....

\begin{tabular}{|c|c|c|c|c|c|c|c|}
\hline Compound \# & $\mathbf{X}$ & Aromatic & $\mathbf{H}_{\mathbf{a}}$ & $\mathbf{H}_{\mathrm{b}}$ & $\mathbf{H}_{\mathrm{c}}$ & $\mathbf{H}_{\mathbf{d}}$ & $\mathbf{X}$ \\
\hline $2 \mathrm{~g}$ & $\mathrm{p}-\mathrm{CH}_{3} \mathrm{CO}$ & $\begin{array}{l}7.91,7.90 \\
7.46,7.44\end{array}$ & $\begin{array}{c}3.86 \\
(\mathrm{t}, 4 \mathrm{H}) \\
\mathrm{J}=5.3\end{array}$ & $\begin{array}{c}2.58 \\
(\mathrm{t}, 4 \mathrm{H}) \\
\mathrm{J}=5.0\end{array}$ & $\begin{array}{c}2.47(\mathrm{q}, 2 \mathrm{H}) \\
\mathrm{J}=7.2\end{array}$ & $\begin{array}{c}1.10(\mathrm{t}, 3 \mathrm{H}) \\
\mathrm{J}=7.2\end{array}$ & $\begin{array}{c}2.55 \\
(\mathrm{~s}, 3 \mathrm{H})\end{array}$ \\
\hline $2 \mathrm{~h}$ & $\mathrm{o}-\mathrm{Br}$ & $\begin{array}{l}7.57(1 \mathrm{H}, \mathrm{dd}) \\
7.41(1 \mathrm{H}, \mathrm{dd}) \\
7.23(1 \mathrm{H}, \mathrm{dt}) \\
7.00(1 \mathrm{H}, \mathrm{dt})\end{array}$ & $\begin{array}{c}3.89 \\
(\mathrm{t}, 4 \mathrm{H}) \\
\mathrm{J}=4.9\end{array}$ & $\begin{array}{c}2.60 \\
(\mathrm{t}, 4 \mathrm{H}) \\
\mathrm{J}=5.1\end{array}$ & $\begin{array}{c}2.49(\mathrm{q}, 2 \mathrm{H}) \\
\mathrm{J}=7.2\end{array}$ & $\begin{array}{c}1.12(\mathrm{t}, 3 \mathrm{H}) \\
\mathrm{J}=7.2\end{array}$ & $\mathrm{n} / \mathrm{a}$ \\
\hline $2 \mathrm{i}$ & pyr & $\begin{array}{c}8.64(1 \mathrm{H}, \mathrm{d}) \\
8.35(1 \mathrm{H}, \mathrm{dd}) \\
7.67(1 \mathrm{H}, \mathrm{dt}) \\
7.20(1 \mathrm{H}, \mathrm{dd})\end{array}$ & $\begin{array}{c}3.82 \\
(\mathrm{t}, 4 \mathrm{H}) \\
\mathrm{J}=5.3\end{array}$ & $\begin{array}{c}2.56 \\
(\mathrm{t}, 4 \mathrm{H}) \\
\mathrm{J}=5.2\end{array}$ & $\begin{array}{c}2.45(\mathrm{q}, 2 \mathrm{H}) \\
\mathrm{J}=7.2\end{array}$ & $\begin{array}{c}1.09(\mathrm{t}, 3 \mathrm{H}) \\
\mathrm{J}=7.2\end{array}$ & $\mathrm{n} / \mathrm{a}$ \\
\hline
\end{tabular}

Table 3. Physical data of 1-(2-hydroxyethyl)-4-[2-aryl-1-diazenyl]piperazines.

\begin{tabular}{|c|c|c|c|c|c|c|c|}
\hline Compound \# & $\mathbf{X}$ & Yield \% & m.p. $\left({ }^{\circ} \mathrm{C}\right)$ & Solvent & Recovery $\%$ & Crystal Appearance & IR $\left(\mathrm{cm}^{-1}\right)$ \\
\hline $3 a$ & $\mathrm{p}-\mathrm{CN}$ & 88 & $157-159$ & Ethanol & 78 & Peach glassy sheets & $\begin{array}{l}2219(\mathrm{CN}) \\
851(\mathrm{OOP})\end{array}$ \\
\hline $3 b$ & $\mathrm{p}-\mathrm{CO}_{2} \mathrm{Me}$ & 93 & $92-93$ & Ethyl Acetate & 49 & Small needles yellow/ brown & $\begin{array}{c}1720(\mathrm{C}=\mathrm{O}) \\
862 / 774(\mathrm{OOP})\end{array}$ \\
\hline $3 c$ & $\mathrm{p}-\mathrm{NO}_{2}$ & 62 & $102-103$ & Methanol & 66 & Amorphous brown & $\begin{array}{c}1509 / 1338\left(\mathrm{NO}_{2}\right) \\
853(\mathrm{OOP})\end{array}$ \\
\hline $3 \mathrm{~d}$ & $\mathrm{p}-\mathrm{CH}_{3}$ & 83 & $69-71$ & Cyclohexane & 84 & Peach coloured sheets & 823 (OOP) \\
\hline $3 \mathrm{e}$ & $\mathrm{p}-\mathrm{Br}$ & 85 & $97-99$ & Cyclohexane & 45 & Light yellow amorphous & $\begin{array}{l}636(\mathrm{C}-\mathrm{Br}) \\
830(\mathrm{OOP})\end{array}$ \\
\hline $3 \mathrm{f}$ & $\mathrm{p}-\mathrm{CH}_{3} \mathrm{O}$ & 74 & $61-62$ & Cyclohexane & 20 & Light brown sheets & $\begin{array}{c}1161 / 1250(\mathrm{C}-\mathrm{O}) \\
832(\mathrm{OOP})\end{array}$ \\
\hline $3 \mathrm{~h}$ & $\mathrm{o}-\mathrm{Br}$ & 100 & $26-27$ & $\mathrm{n} / \mathrm{a}$ & $\mathrm{n} / \mathrm{a}$ & Orange amorphous & 649/689 (C-Br) \\
\hline $3 \mathrm{i}$ & pyr & 86 & Oil & $\mathrm{n} / \mathrm{a}$ & $\mathrm{n} / \mathrm{a}$ & Red spikes growing up vial & $1665 / 1589(\mathrm{C}=\mathrm{N})$ \\
\hline
\end{tabular}

Table 4. ${ }^{1} \mathrm{H}$ NMR chemical shift data (ppm) in $\mathrm{CDCl}_{3}$ of the 1-(2-hydroxyethyl)-4-[2-aryl-1-diazenyl]piperazines.

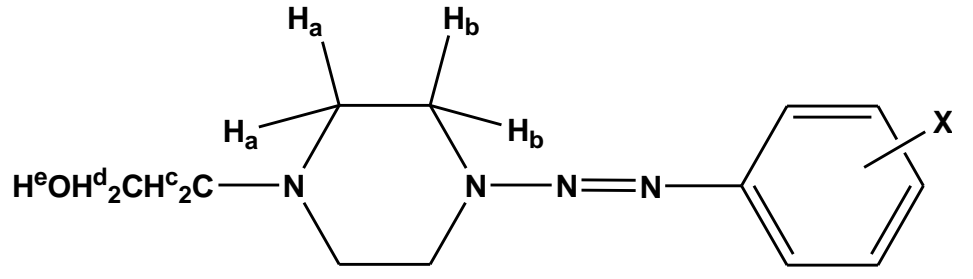

\begin{tabular}{|c|c|c|c|c|c|c|c|c|}
\hline Compound \# & $\mathbf{X}$ & Aromatic & $\mathbf{H}_{\mathbf{a}}$ & $\mathbf{H}_{\mathbf{b}}$ & $\mathbf{H}_{\mathbf{c}}$ & $\mathbf{H}_{\mathbf{d}}$ & $\mathbf{H}_{\mathbf{e}}$ & $\mathbf{X}$ \\
\hline \hline \multirow{2}{*}{ 3a } & $\mathrm{p}-\mathrm{CN}$ & $7.63,7.60$, & 3.91 & 2.67 & 2.65 & 3.69 & 1.8 & $\mathrm{n} / \mathrm{a}$ \\
& & $7.51,7.47$ & $(\mathrm{t}, 4 \mathrm{H})$ & $(\mathrm{t}, 4 \mathrm{H})$ & $(\mathrm{t}, 2 \mathrm{H})$ & $(\mathrm{t}, 2 \mathrm{H})$ & $(\mathrm{s}, 1 \mathrm{H})$ \\
& & & $\mathrm{J}=5.4$ & $\mathrm{~J}=5.6$ & $\mathrm{~J}=5.4$ & $\mathrm{~J}=5.3$ & $\mathrm{v} . \mathrm{broad}$ & \\
\hline
\end{tabular}


Table 4. Contd.....

\begin{tabular}{|c|c|c|c|c|c|c|c|c|}
\hline Compound \# & $\mathbf{x}$ & Aromatic & $\mathbf{H}_{\mathbf{a}}$ & $\mathbf{H}_{\mathrm{b}}$ & $\mathbf{H}_{\mathbf{c}}$ & $\mathbf{H}_{\mathrm{d}}$ & $\mathbf{H}_{\mathrm{e}}$ & $\mathbf{X}$ \\
\hline $3 b$ & $\mathrm{p}-\mathrm{CO}_{2} \mathrm{Me}$ & $\begin{array}{l}8.04,8.00 \\
7.49,7.45\end{array}$ & $\begin{array}{c}3.88 \\
(\mathrm{t}, 4 \mathrm{H}) \\
\mathrm{J}=5.5\end{array}$ & $\begin{array}{c}2.67 \\
(\mathrm{t}, 4 \mathrm{H}) \\
\mathrm{J}=5.6\end{array}$ & $\begin{array}{c}2.64 \\
(\mathrm{t}, 2 \mathrm{H}) \\
\mathrm{J}=5.4\end{array}$ & $\begin{array}{c}3.68 \\
(\mathrm{t}, 2 \mathrm{H}) \\
\mathrm{J}=5.4\end{array}$ & $\begin{array}{c}1.76 \text { (broad } \\
\mathrm{s}, 1 \mathrm{H})\end{array}$ & $\begin{array}{c}3.90 \\
(\mathrm{~s}, 3 \mathrm{H})\end{array}$ \\
\hline $3 c$ & $\mathrm{p}-\mathrm{NO}_{2}$ & $\begin{array}{l}8.23,8.20 \\
7.55,7.51\end{array}$ & $\begin{array}{c}3.96 \\
\text { (broad t, 4H) } \\
\mathrm{J}=3.8\end{array}$ & $\begin{array}{c}2.68 \\
(\text { broad t, } 4 \mathrm{H}) \\
\mathrm{J}=5.0\end{array}$ & $\begin{array}{c}2.66 \\
\text { (broad t, 2H) }\end{array}$ & $\begin{array}{c}3.72 \\
\text { (broad t, 2H) } \\
\mathrm{J}=5.0\end{array}$ & $\begin{array}{c}1.3 \\
\text { (broad s, } \\
1 \mathrm{H})\end{array}$ & $\mathrm{n} / \mathrm{a}$ \\
\hline $3 \mathrm{~d}$ & $\mathrm{p}-\mathrm{CH}_{3}$ & $\begin{array}{l}7.36,7.33 \\
7.17,7.13\end{array}$ & $\begin{array}{c}3.79 \\
(\mathrm{t}, 4 \mathrm{H}) \\
\mathrm{J}=5.3\end{array}$ & $\begin{array}{c}2.68 \\
(\mathrm{t} .4 \mathrm{H}) \\
\mathrm{J}=5.4\end{array}$ & $\begin{array}{c}2.63 \\
(\mathrm{t}, 2 \mathrm{H}) \\
\mathrm{J}=5.6\end{array}$ & $\begin{array}{c}3.67 \\
(t, 2 \mathrm{H}) \\
\mathrm{J}=5.3\end{array}$ & $\begin{array}{c}1.66 \\
\text { (broad s, } \\
1 \mathrm{H})\end{array}$ & $\begin{array}{c}2.34 \\
(\mathrm{~s}, 3 \mathrm{H})\end{array}$ \\
\hline $3 e$ & $\mathrm{p}-\mathrm{Br}$ & $\begin{array}{l}7.47,7.44 \\
7.33,7.30\end{array}$ & $\begin{array}{c}3.82 \\
(\mathrm{t}, 4 \mathrm{H}) \\
\mathrm{J}=5.3\end{array}$ & $\begin{array}{c}2.68 \\
(\mathrm{t}, 4 \mathrm{H}) \\
\mathrm{J}=5.1\end{array}$ & $\begin{array}{c}2.64 \\
(\mathrm{t}, 2 \mathrm{H}) \\
\mathrm{J}=5.1\end{array}$ & $\begin{array}{c}3.68 \\
(\mathrm{t}, 2 \mathrm{H}) \\
\mathrm{J}=5.1\end{array}$ & $\begin{array}{c}1.65 \\
\text { (broad s, } \\
1 \mathrm{H})\end{array}$ & $\mathrm{n} / \mathrm{a}$ \\
\hline $3 \mathrm{f}$ & $\mathrm{p}-\mathrm{CH}_{3} \mathrm{O}$ & $\begin{array}{l}7.43,7.40 \\
6.90,6.87\end{array}$ & $\begin{array}{c}3.76 \\
(\mathrm{t}, 4 \mathrm{H}) \\
\mathrm{J}=5.3\end{array}$ & $\begin{array}{c}2.67 \\
(\mathrm{t}, 4 \mathrm{H}) \\
\mathrm{J}=5.5\end{array}$ & $\begin{array}{c}2.63 \\
(\mathrm{t}, 2 \mathrm{H}) \\
\mathrm{J}=5.4\end{array}$ & $\begin{array}{c}3.67 \\
(\mathrm{t}, 2 \mathrm{H}) \\
\mathrm{J}=5.3\end{array}$ & $\begin{array}{c}1.76 \\
\text { (v. broad s, } \\
1 \mathrm{H})\end{array}$ & $\begin{array}{c}3.82 \\
(\mathrm{~s}, 3 \mathrm{H})\end{array}$ \\
\hline $3 g$ & $\mathrm{p}-\mathrm{CH}_{3} \mathrm{CO}$ & $\begin{array}{l}7.97,7.94 \\
7.51,7.47\end{array}$ & $\begin{array}{c}3.89 \\
(\mathrm{t}, 4 \mathrm{H}) \\
\mathrm{J}=5.1\end{array}$ & $\begin{array}{c}2.69 \\
(\mathrm{t}, 4 \mathrm{H}) \\
\mathrm{J}=5.3\end{array}$ & $\begin{array}{c}2.64 \\
(\mathrm{t}, 2 \mathrm{H}) \\
\mathrm{J}=5.4\end{array}$ & $\begin{array}{c}3.69 \\
(\mathrm{t}, 2 \mathrm{H}) \\
\mathrm{J}=5.1\end{array}$ & $\begin{array}{c}1.7 \\
\text { (broad s, } \\
1 \mathrm{H})\end{array}$ & $\begin{array}{c}2.59 \\
(\mathrm{~s}, 3 \mathrm{H})\end{array}$ \\
\hline $3 \mathrm{~h}$ & $\mathrm{o}-\mathrm{Br}$ & $\begin{array}{l}7.59(1 \mathrm{H}, \mathrm{dd}) \\
7.42(1 \mathrm{H}, \mathrm{dd}) \\
7.26(1 \mathrm{H}, \mathrm{dt}) \\
7.02(1 \mathrm{H}, \mathrm{dt})\end{array}$ & $\begin{array}{c}3.89(\mathrm{t}, 4 \mathrm{H}) \\
\mathrm{J}=4.9\end{array}$ & $\begin{array}{c}2.69 \\
(\mathrm{t}, 4 \mathrm{H}) \\
\mathrm{J}=5.13\end{array}$ & $\begin{array}{c}2.64 \\
(\mathrm{t}, 2 \mathrm{H}) \\
\mathrm{J}=5.4\end{array}$ & $\begin{array}{c}3.68 \\
(\mathrm{t}, 2 \mathrm{H}) \\
\mathrm{J}=5.0\end{array}$ & $\begin{array}{c}1.77 \\
\text { (broad s, } \\
1 \mathrm{H})\end{array}$ & $\mathrm{n} / \mathrm{a}$ \\
\hline
\end{tabular}

Table 5. Physical data of 1-butyl-4-[2-aryl-1-diazenyl]piperazines.

\begin{tabular}{|c|c|c|c|c|c|c|c|}
\hline Compound \# & $\mathbf{X}$ & Yield \% & m.p. $\left({ }^{\circ} \mathbf{C}\right)$ & Solvent & Recovery \% & State of Crystal \\
\hline \hline $4 \mathrm{a}$ & $\mathrm{p}-\mathrm{CN}$ & 24 & $41-42$ & $\mathrm{n} / \mathrm{a}$ & $\mathrm{n} / \mathrm{a}$ & Amorphous light brown & $\begin{array}{c}2216.6(\mathrm{CN}) \\
846.8(\mathrm{OOP})\end{array}$ \\
\hline $4 \mathrm{~b}$ & $\mathrm{p}-\mathrm{CO}_{2} \mathrm{CH}_{3}$ & 67 & $82-83$ & Ethanol & 26 & White glassy, possibly in sheets & $\begin{array}{c}1718.9(\mathrm{C}=\mathrm{O}) \\
865(\mathrm{OOP})\end{array}$ \\
\hline $4 \mathrm{c}$ & $\mathrm{p}-\mathrm{Br}$ & 86 & $52-54$ & Ethanol & 20 & Some white and some brown sheets & $\begin{array}{c}637(\mathrm{C}-\mathrm{Br}) \\
834(\mathrm{OOP})\end{array}$ \\
\hline $4 \mathrm{~d}$ & $\mathrm{p}-\mathrm{CH}_{3} \mathrm{O}$ & 14 & $30-32$ & $\mathrm{n} / \mathrm{a}$ & $\mathrm{n} / \mathrm{a}$ & Amorphous copper colour & $\begin{array}{c}1246\left(\mathrm{CH} \mathrm{O}_{3}\right) \\
836(\mathrm{OOP})\end{array}$ \\
\hline
\end{tabular}


Table 6. ${ }^{1} \mathrm{H}$ NMR chemical shift data (ppm) in $\mathrm{CDCl}_{3}$ of 1-butyl-4-[2-aryl-1-diazenyl]piperazines.

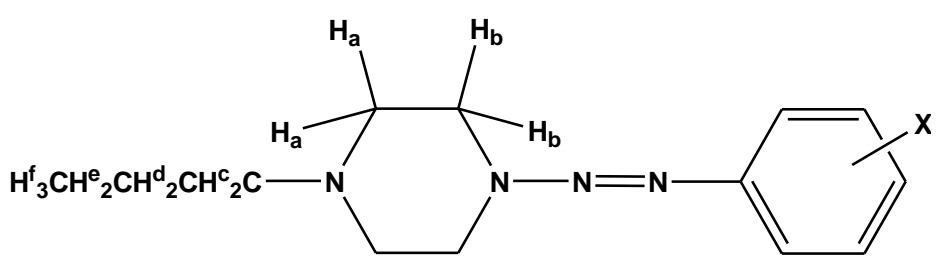

\begin{tabular}{|c|c|c|c|c|c|c|c|c|c|}
\hline Compound \# & $\mathbf{X}$ & Aromatic & $\mathbf{H}_{\mathbf{a}}$ & $\mathbf{H}_{\mathrm{b}}$ & $\mathbf{H}_{\mathrm{c}}$ & $\mathbf{H}_{\mathbf{d}}$ & $\mathbf{H}_{\mathrm{e}}$ & $\mathbf{H}_{\mathrm{f}}$ & $\mathbf{X}$ \\
\hline $4 a$ & $\mathrm{p}-\mathrm{CN}$ & $\begin{array}{l}7.637 .59 \\
7.507 .47\end{array}$ & $\begin{array}{c}3.90 \\
(\mathrm{t}, 4 \mathrm{H}) \\
\mathrm{J}=5.3\end{array}$ & $\begin{array}{c}2.59 \\
(\mathrm{t}, 4 \mathrm{H}) \\
\mathrm{J}=4.9\end{array}$ & $\begin{array}{c}2.42 \\
(\mathrm{t}, 2 \mathrm{H}) \\
\mathrm{J}=7.5\end{array}$ & $\begin{array}{c}1.52 \\
(\mathrm{p}, 2 \mathrm{H}) \\
\mathrm{J}=7.8\end{array}$ & $\begin{array}{c}1.37 \\
(\mathrm{p}, 2 \mathrm{H}) \\
\mathrm{J}=7.3\end{array}$ & $\begin{array}{c}0.94 \\
(\mathrm{t}, 3 \mathrm{H}) \\
\mathrm{J}=7.0\end{array}$ & $\mathrm{n} / \mathrm{a}$ \\
\hline $4 b$ & $\mathrm{p}-\mathrm{CO}_{2} \mathrm{Me}$ & $\begin{array}{l}8.038 .00 \\
7.497 .45\end{array}$ & $\begin{array}{c}3.88 \\
(\mathrm{t}, 4 \mathrm{H}) \\
\mathrm{J}=5.5\end{array}$ & $\begin{array}{c}2.60 \\
(\mathrm{t}, 4 \mathrm{H}) \\
\mathrm{J}=5.1\end{array}$ & $\begin{array}{c}2.42 \\
(\mathrm{t}, 2 \mathrm{H}) \\
\mathrm{J}=7.6\end{array}$ & $\begin{array}{c}1.52 \\
(\mathrm{p}, 2 \mathrm{H}) \\
\mathrm{J}=7.6\end{array}$ & $\begin{array}{c}1.35 \\
(\mathrm{p}, 2 \mathrm{H}) \\
\mathrm{J}=7.3\end{array}$ & $\begin{array}{c}0.94 \\
(\mathrm{t}, 3 \mathrm{H}) \\
\mathrm{J}=7.3\end{array}$ & $\begin{array}{c}3.90 \\
(\mathrm{~s}, 3 \mathrm{H})\end{array}$ \\
\hline $4 \mathrm{c}$ & $\mathrm{p}-\mathrm{Br}$ & $\begin{array}{l}7.477 .43 \\
7.337 .29\end{array}$ & $\begin{array}{c}3.81 \\
(\mathrm{t}, 4 \mathrm{H}) \\
\mathrm{J}=5.0\end{array}$ & $\begin{array}{c}2.59 \\
(\mathrm{t}, 4 \mathrm{H}) \\
\mathrm{J}=5.0\end{array}$ & $\begin{array}{c}2.41 \\
(\mathrm{t}, 2 \mathrm{H}) \\
\mathrm{J}=7.5\end{array}$ & $\begin{array}{c}1.52 \\
(\mathrm{p}, 2 \mathrm{H}) \\
\mathrm{J}=7.5\end{array}$ & $\begin{array}{c}1.36 \\
(\mathrm{p}, 2 \mathrm{H}) \\
\mathrm{J}=7.5\end{array}$ & $\begin{array}{c}0.94 \\
(\mathrm{t}, 3 \mathrm{H}) \\
\mathrm{J}=7.5\end{array}$ & $\mathrm{n} / \mathrm{a}$ \\
\hline
\end{tabular}

Table 7. Physical data of 1-isopropyl-4-[2-aryl-1-diazenyl]piperazines.

\begin{tabular}{|c|c|c|c|c|c|}
\hline Compound \# & $\mathbf{X}$ & Yield \% & m.p. $\left({ }^{\circ} \mathbf{C}\right)$ & State of Crystal & IR \\
\hline \hline $5 \mathrm{a}$ & $\mathrm{p}-\mathrm{CN}$ & 84 & $57-59$ & Amorphous light brown & $\begin{array}{c}2221.2(\mathrm{CN}) \\
846.9(\mathrm{OOP})\end{array}$ \\
\hline $5 \mathrm{~b}$ & $\mathrm{p}-\mathrm{CO}_{2} \mathrm{Me}$ & 85 & $61-62$ & Amorphous red/ brown & $\begin{array}{c}1716.6(\mathrm{C}=\mathrm{O}) \\
821.2(\mathrm{OOP})\end{array}$ \\
\hline
\end{tabular}

Table 8. ${ }^{1} \mathrm{H}$ NMR chemical shift data (ppm) in $\mathrm{CDCl}_{3}$ 1-isopropyl-4-[2-aryl-1-diazenyl]piperazine.

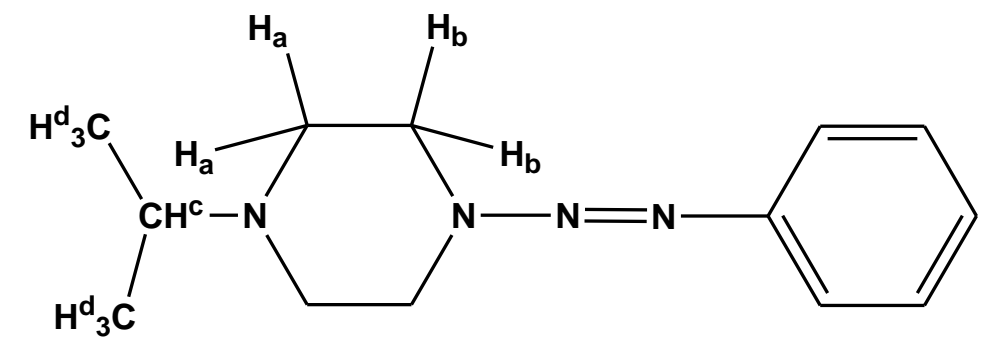

\begin{tabular}{|c|c|c|c|c|c|c|c|}
\hline Compound \# & $\mathbf{X}$ & Aromatic & $\mathbf{H}_{\mathbf{a}}$ & $\mathbf{H}_{\mathbf{a}}$ & $\mathbf{H}_{\mathrm{c}}$ & $\mathbf{H}_{\mathbf{d}}$ & $\mathbf{X}$ \\
\hline $5 b$ & $\mathrm{p}-\mathrm{CO}_{2} \mathrm{Me}$ & $\begin{array}{l}8.01,7.99 \\
7.46,7.45\end{array}$ & $\begin{array}{c}3.86 \\
(\mathrm{t}, 4 \mathrm{H}) \\
\mathrm{J}=5.2\end{array}$ & $\begin{array}{c}2.67 \\
(\mathrm{t}, 4 \mathrm{H}) \\
\mathrm{J}=5.0\end{array}$ & $\begin{array}{c}2.81 \\
\text { (septet, } 1 \mathrm{H} \text { ) } \\
\mathrm{J}=6.6\end{array}$ & $\begin{array}{c}1.075 \\
(\mathrm{~d}, 6 \mathrm{H}) \\
\mathrm{J}=6.8\end{array}$ & $\begin{array}{c}3.89 \\
(\mathrm{~s}, 3 \mathrm{H})\end{array}$ \\
\hline
\end{tabular}


Table 9. High Resolution Mass Spectrometric Analysis of the 1-alkyl-4-[2-aryl-1-diazenyl]piperazines.

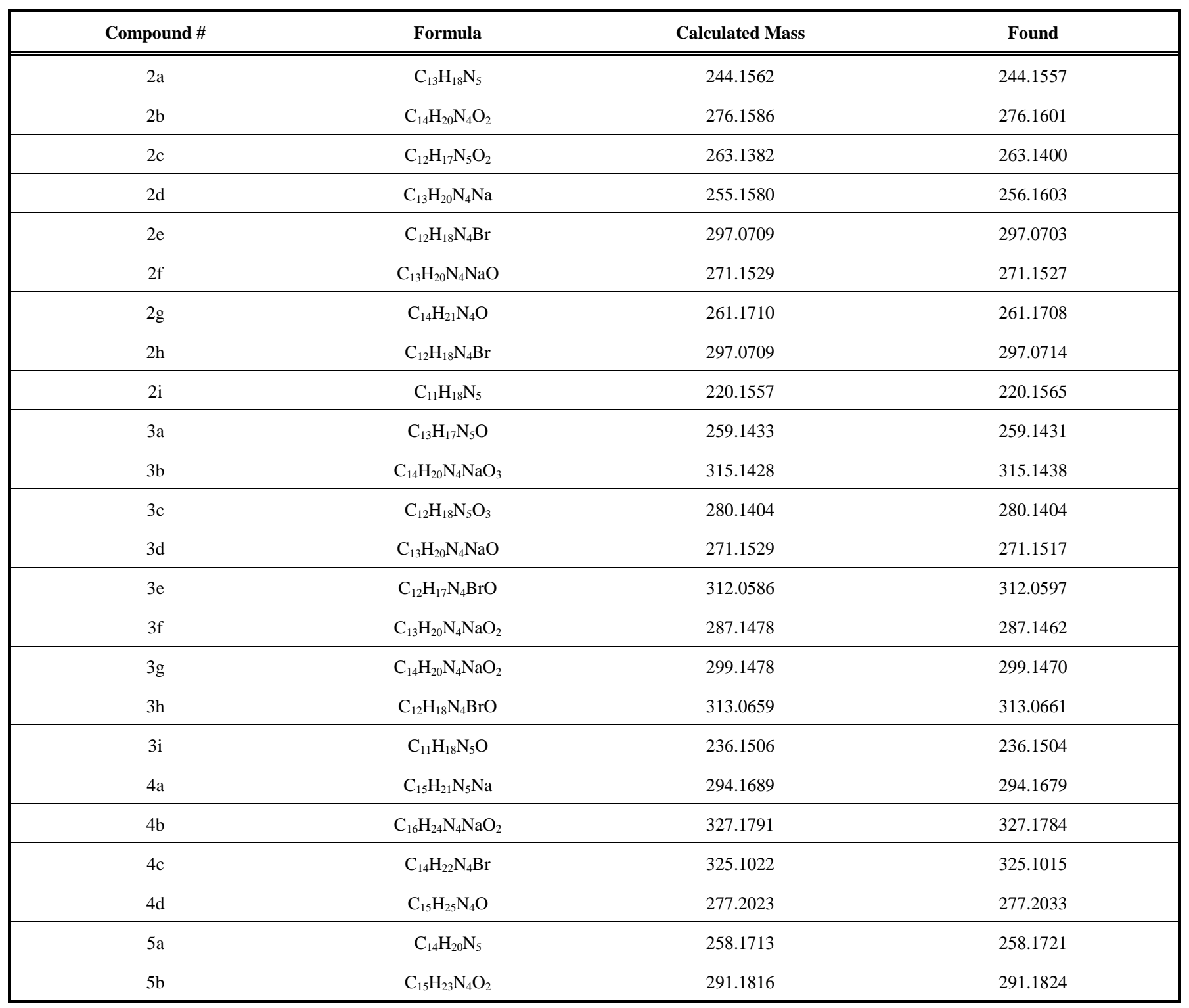

\section{EXPERIMENTAL}

The general techniques of measuring physical properties can be found in a number of our previous papers $[1,3,4,6$, 7].

\section{1-Alkyl-4-[2-aryl-1-diazenyl]piperazine}

\section{General Procedure}

The aromatic primary amine $(0.01 \mathrm{~mol})$ was dissolved in $10 \mathrm{~mL}$ of $3 \mathrm{~mol} / \mathrm{L} \mathrm{HCl}$ and $5 \mathrm{~mL}$ of water, with the aid of heat if necessary. The resulting solution was cooled in an ice/salt bath to $0^{\circ} \mathrm{C}$. The solution was diazotized with a solution of sodium nitrite $(0.011 \mathrm{~mol}, 0.76 \mathrm{~g})$ in water $10 \mathrm{~mL}$, while maintaining that the temperature did not exceed $5^{\circ} \mathrm{C}$, and then stirred for $30 \mathrm{~min}$ in the cold, after which, the 1alkylpiperazine $(0.10 \mathrm{~mol})$ in $2 \mathrm{~mL}$ water was added drop wise while still keeping the temperature below $5^{\circ} \mathrm{C}$. The solution was neutralized with a saturated aqueous solution of sodium bicarbonate, and then left to stir in the cold for a further 30 min until precipitation was deemed complete. The solid product was separated by suction filtration, dried, and recrystallized from an appropriate solvent. In some cases the product was an oil, in which case separation was achieved with an extraction performed with dichloromethane.

\section{CONCLUSION}

The results reported here provide a comprehensive account of the synthesis and characterization of the 1-ethyl (2), 1-(2-hydroxyethyl) (3), 1-butyl (4) and 1-isopropyl-4-[2aryl-1-diazenyl]piperazines (5). This will prove to be a valuable resource of data for structure elucidation of related compounds.

\section{CONFLICT OF INTEREST}

The authors confirm that this article content has no conflict of interest. 


\section{ACKNOWLEDGEMENTS}

The authors are grateful to the Natural Sciences and Engineering Research Council of Canada (NSERC) for a Discovery Grant to the principal author $(\mathrm{KV})$. We are also grateful to the Faculty of Graduate Studies and Research at Saint Mary's University for a Summer Research Award (2010). We are also grateful to the Atlantic Region Magnetic Resonance Centre at Dalhousie University for providing NMR spectra, and to Dalhousie University for providing mass spectral data. In particular, we would like to thank Dr. Mike Lumsden for assistance with the NMR spectral data, and Mr. Xiao Feng for assistance with mass spectra.

\section{REFERENCES}

[1] Little, V.R.; Vaughan, K. Synthesis and characterization of a series of 1-methyl-4-[2-aryl-1-diazenyl]piperazines and a series of ethyl 4-[2-aryl-1-diazenyl]-1-piperazinecarboxylates. Can. J. Chem., 2004, 82, 1294-1303.
[2] Little, V.R.; Jenkins, H.; Vaughan, K. An x-ray crystallographic study of the related triazenes, 1,4-di[ $[(E)-2$-(2-nitrophenyl)-1diazenyl]piperazine and methyl 4-\{(E)-2-(4-methylpiperazino)-1diazenyl \}benzoate. J. Chem. Cryst., 2008, 38, 447-452.

[3] Moser, S.L.; Vaughan, K. Synthesis and characterization of a series of 4-methyl-1-2-aryl-1-diazenyl]-1,4-diazepanes and 1,4-di-[2-aryl1-diazenyl]-1,4-diazepanes. Can. J. Chem., 2004, 82, 1725-1735.

[4] Little, V.R.; Tingley, R.; Vaughan, K. Triazene derivatives of $[1, \mathrm{x}]$-diazacycloalkanes. Part III. Synthesis and characterization of a series of 1,4-di[2-aryl-1-diazenyl]piperazines. Can. J. Chem., 2005, 83, 471-476.

[5] Yanarates, E.; Disli, A.; Yildirir. Y. New N,N'-bis-[substituted phenylazo]piperazines and their cleavage reactions in acetic acid Org. Prep. Proc. Int., 1999, 31, 429-433.

[6] Hunter, N.; Tingley, R.; Peori, M.B.; Vaughan, K. Triazene derivatives of $[1, \mathrm{x}]$-diazacycloalkanes. Part VIII. Synthesis and characterization of a series of 1,4-di[2-aryl-1-diazenyl]-2methylpiperazines. Can. J. Chem., 2007 85, 189-196.

[7] Hunter, N.; Vaughan, K. Triazene derivatives of $[1, \mathrm{x}]-$ diazacycloalkanes. Part IX. Synthesis and characterization of a series of 1,4-di[2-aryl-1-diazenyl]-2,6-dimethylpiperazines. Can. J. Chem., 2010, 88, 344-351.

Received: August 12, 2014

Revised: December 08, 2014

Accepted: December 11, 2014

(C) MacLeod and Vaughan; Licensee Bentham Open.

This is an open access article licensed under the terms of the Creative Commons Attribution Non-Commercial License (http://creativecommons.org/licenses/ by-nc/3.0/) which permits unrestricted, non-commercial use, distribution and reproduction in any medium, provided the work is properly cited. 